\title{
Estudo comparativo da eficácia decolírios antiinflamatórios não esteróides na manutenção da dilatação pupilar durante a cirurgia de catarata
}

\author{
Comparative efficacyof nonsteroidal antiinflammatory eyedrops in maintenance of \\ pupillary dilation during cataractsurgery
}

Rafael Naves Tomás ${ }^{1}$

Lúcio Moraes ${ }^{2}$

Humberto Borges da Silva ${ }^{3}$

José Beniz Neto ${ }^{4}$

Marcos Ávila ${ }^{5}$

\footnotetext{
Trabalho realizado no Centro de Referência em Oftalmologia do Hospital de Clínicas da Universidade Federal de Goiás - CEROF.

${ }^{1}$ Acadêmico do $5^{\circ}$ ano e monitor do serviço de Oftalmologia da Faculdade de Medicina da Universidade Federal de Goiás.

${ }^{2}$ Médico Oftalmologista do Centro de Referência em Oftalmologia - CEROF do Hospital das Clínicas da Faculdade de Medicina da Universidade Federal de Goiás.

${ }^{3}$ Médico residente do $3^{\circ}$ ano em Oftalmologia do Centro de Referência em Oftalmologia do Hospital de Clínicas da Faculdade de Medicina da Universidade Federal de Goiás - CEROF.

${ }^{4}$ Médico Oftalmologista do Centro de Referência em Oftalmologia - CEROF do Hospital das Clínicas da Faculdade de Medicina da Universidade Federal de Goiás.

Professor e chefe do serviço de Oftalmologia e Centro

de Referência em Oftalmologia - CEROF do Hospital das Clínicas da Faculdade de Medicina da Universidade Federal de Goiás.

Endereço para correspondência: Rafael Naves Tomás Rua 3, no 222/202 - Ed. Ademar - Setor Oeste.

Goiânia (GO) CEP 74110-130

e-mail: navesrafael@hotmail.com

Recebido para análise em 03.06.2002

Versão revisada recebida em 30.11.2002

Aprovação em 07.07.2003
}

\section{RESUMO}

Objetivo: Antiinflamatórios não hormonais, com efeitos antiprostaglandínicos, têm sido usados no pré-operatório de cirurgia de catarata para manutenção da midríase per-operatória. Infelizmente, ainda não existe um antiprostaglandínico ideal. Este é um estudo de corte transversal, randomizado, grupo-placebo e duplo-mascarado, comparando a eficácia do cetorolac de trometamina, flurbiprofeno sódico, diclofenaco sódico e um placebo, hipromelose, quanto à manutenção peroperatória da midríase. Métodos: Cento e dezesseis pacientes, submetidos à extração extracapsular da catarata com implantação de lente intra-ocular, foram separados aleatoriamente em quatro grupos, que receberam a mesma rotina de dilatação e de instilação dos antiprostaglandínicos. O grupo I recebeu cetorolac de trometamina; grupo II, flurbiprofeno sódico; grupo III, diclofenaco sódico e grupo IV, hipromelose, o placebo. Os dados referentes à pupila foram obtidos por meio de questionário objetivo proposto aos cirurgiões no início e ao final da cirurgia. Resultados: Os resultados mostraram diferença significativa entre o grupo I e o placebo quanto à manutenção da dilatação peroperatória. Também foi significativa a diferença entre o grupo I e os demais grupos em relação à suficiência da midríase para o início do ato cirúrgico e para a ocorrência de miose intra-operatória. Não se registrou diferença significante entre o uso de flurbiprofeno e de diclofenaco quando comparados ao placebo para os mesmos parâmetros $(\mathrm{p}<0,05)$. Conclusões: Os resultados mostraram que o cetorolac foi mais eficiente do que os medicamentos utilizados nos demais grupos em relação aos parâmetros testados. Embora se registrem diferenças percentuais entre os resultados alcançados com flurbiprofeno e diclofenaco as diferenças não são estatisticamente significativas. Estudos feitos sobre amostra maior e/ou estudos multicêntricos poderão definir com clareza o papel definitivo dos antiinflamatórios não hormonais na manutenção da midríase peroperatória.

Descritores: Soluções oftálmicas; Extração de catarata; Pupila/efeito de drogas; Antiinflamatórios não-esteróide/uso terapêutico; Eficácia; Estudo comparativo; Adulto

\section{INTRODUÇÃO}

É importante para o cirurgião contar com a melhor dilatação pupilar possível durante a cirurgia de catarata. A miose intra-operatória, muitas vezes provocada pela liberação de prostaglandinas, é atribuída à manipulação da íris ou ao preparo pré-operatório inadequado e pode gerar obstáculos durante a cirurgia, tais como dificuldades na aspiração cortical, aumento 
820 Estudo comparativo da eficácia de colírios antiinflamatórios não esteróides na manutenção da dilatação pupilar durante a cirurgia de catarata

na incidência de ruptura de cápsula posterior e dificuldades no implante da lente intra-ocular(LIO) ${ }^{(1)}$.

Alguns antiinflamatórios não-esteróides (AINEs) de uso ocular vêm sendo estudados com o objetivo de manutenção da midríase no per-operatório. O diclofenaco sódico é um derivado do ácido fenilacético e o flurbiprofeno, do ácido propiônico ${ }^{(2-4)}$. As duas drogas inibem a produção de prostaglandinas e tromboxanos através da inibição da ação da enzima ciclo-oxigenase sobre o ácido aracdônico por um mecanismo competitivo, tempo-dependente e irreversível. Além disso, o diclofenaco ainda reduz a produção de leucotrienos in vitro desviando parcialmente o ácido aracdônico e os triglicerídeos ${ }^{(5-6)}$.

O cetorolac de trometamina é um derivado pirrolo-pirrólico do ácido carboxílico. Seu efeito analgésico é comparável ao da morfina sem, entretanto, seu efeito narcotizante ou potencial para causar dependência. É também capaz de inibir a enzima ciclo-oxigenase, provocando a redução dos níveis de prostaglandina no humor aquoso, especialmente da prostaglandina $\mathrm{E}_{2}\left(\mathrm{PGE}_{2}\right)^{(7)}$.

A hipromelose está presente em solução oftálmica coloidal que se identifica com a fisiologia ocular e substitui a lágrima, agindo como lubrificante dos tecidos oculares sem efeitos colaterais significativos.

A maioria das informações disponibilizadas até o presente vem da literatura internacional e compara, isoladamente, duas drogas e um placebo ${ }^{(4-8)}$. Não se encontrou registro, no Brasil, de estudo comparativo entre as drogas aqui estudadas (incluindo o placebo) na manutenção da dilatação pupilar em pacientes submetidos à cirurgia de catarata.

Este estudo objetiva comparar entre si e com um placebo a ação dos três principais agentes antiinflamatórios utilizados no Brasil como colírios na obtenção de midríase per-operatória na cirurgia de catarata, buscando identificar aquele com efeito mais evidente sobre os resultados cirúrgicos esperados. Foram comparados o cetorolac de trometamina, o flurbiprofeno sódico e o diclofenaco sódico frente a um placebo, a hipromelose.

\section{MÉTODOS}

Este é um estudo randomizado, duplo-mascarado, de corte transversal e com grupo placebo sobre o efeito do cetorolac, flurbiprofeno e diclofenaco, comparados à hipromelose, sobre a manutenção per-operatória da dilatação pupilar na cirurgia de catarata. É um estudo que segue os princípios da Declaração de Helsinque e foi aprovado pelo comitê de Ética do Hospital das Clínicas da Universidade Federal de Goiás.

Cento e dezesseis pacientes, após assinarem o consentimento livre e esclarecido, foram submetidos à extração extracapsular da catarata. Foram excluídos do estudo pacientes em tratamento para glaucoma, submetidos à cirurgia recente em qualquer dos olhos, em tratamento com drogas anticolinérgicas, com história de ceratite por Herpes simplex, sangramentos oculares, intolerância a drogas antiinflamatórias não-esteróides, em terapia concomitante com uso de AINEs, com de- sordens pupilares que pudessem afetar o estudo, com doença inflamatória ocular, uveíte anterior cicatrizada ou úlcera péptica $^{(6-9)}$.

Os pacientes incluídos no estudo foram aleatoriamente divididos em 4 grupos, com administração de cetorolac de trometamina $0,5 \%$ (grupo I), flurbiprofeno sódico $0,03 \%$ (grupo II), diclofenaco sódico 0,1\% (grupo III) e hipromelose (grupo IV). Em todos os casos foi seguida a rotina de quatro instilações a intervalos de 15 minutos, iniciadas uma hora e meia antes da cirurgia. Utilizaram-se os colírios disponíveis comercialmente, em sua apresentação original, ficando estabelecida a rotina de dilatação com três instilações a cada 10 minutos durante a primeira hora anterior à cirurgia, utilizando solução midriática contendo tropicamida $5 \%$ e fenilefrina $1 \%{ }^{(10)}$ na proporção de $50 \%$ de cada uma das drogas. Estevese atento para que os cirurgiões não tivessem conhecimento sobre as drogas instiladas no preparo pré-operatório.

Avaliou-se o tamanho pupilar no início e ao final de cada cirurgia, apontando-o no instrumento padronizado entregue ao cirurgião e que contém outros dados relativos ao paciente. Os cirurgiões classificaram o tamanho pupilar em categorias: grande (maior que $6 \mathrm{~mm}$ ), médio (entre 3 e $6 \mathrm{~mm}$ ) ou pequeno (entre 2 e $3 \mathrm{~mm}$ ). Investigou-se ainda se a midríase foi suficiente $(>6 \mathrm{mmm})$ para a cirurgia, se houve miose $(<3 \mathrm{mmm})$ e/ou necessidade do uso de adrenalina intra-operatória para aumento da midríase e a resposta ao carbacol no fechamento pupilar ao final da cirurgia.

As cirurgias foram realizadas por médicos professores, contratados e residentes do $3^{\circ}$ ano ${ }^{(10)}$ do Hospital das Clínicas da Universidade Federal de Goiás, com a seguinte técnica cirúrgica uniforme: incisão escleral tunelizada, capsulorrexe, expressão do núcleo, remoção de massas, colocação de lente intra-ocular em câmara posterior, sutura do túnel e conjuntival, injeção de carbacol em câmara anterior e injeção subconjuntival de garamicina e decadron.

Avaliou-se o efeito das drogas (e do placebo) sobre a pupila e sua associação com outras variáveis (sexo e idade), utilizando-se testes estatísticos: teste do qui-quadrado, t-student, análise de variância e estatística descritiva. Aceitou-se um valor de $p<0,05$ como significativo.

\section{RESULTADOS}

Os 116 pacientes que compuseram a amostra não exibiram diferença estatisticamente significativa em relação ao sexo nos quatro grupos. E as suas idades variaram de 23 a 86 anos, com médias entre 65,80 no grupo II $(+10,36)$ e $68,54(+9,58)$ no grupo III, também não se registrando diferenças significativas entre os quatro grupos estudados.

Comparou-se o desempenho dos colírios na manutenção da dilatação pupilar per-operatória, e verificou-se que a dilatação no grupo I (cetorolac) se manteve em 52,77\% dos casos, contra 26,92\% no grupo II (flurbiprofeno sódico), 39,39\% no grupo III (diclofenaco sódico) e 19,04\% no grupo IV ou 


\begin{tabular}{|c|c|c|c|c|c|c|c|c|c|c|}
\hline \multirow[b]{2}{*}{ i } & \multirow[b]{2}{*}{ j } & \multicolumn{3}{|c|}{ Grupo i } & \multicolumn{3}{|c|}{ Grupo j } & \multirow[b]{2}{*}{$x^{2}$} & \multirow[b]{2}{*}{$p$} & \multirow[b]{2}{*}{ Significância } \\
\hline & & $\overline{n^{*}}$ & $\mathbf{k}^{\star \star}$ & $\%$ & $n^{*}$ & $\mathbf{k}^{\star \star}$ & $\%$ & & & \\
\hline I & II & 36 & 19 & 52,8 & 26 & 7 & 26,9 & 4,151 & 0,125 & NS \\
\hline 1 & III & 36 & 19 & 52,8 & 33 & 13 & 39,4 & 1,254 & 0,534 & NS \\
\hline I & IV & 36 & 19 & 52,8 & 21 & 4 & 19,0 & 6,261 & 0,044 & S \\
\hline II & III & 26 & 7 & 26,9 & 33 & 13 & 39,4 & 1,011 & 0,603 & NS \\
\hline II & IV & 26 & 7 & 26,9 & 21 & 4 & 19,0 & 0,408 & 0,815 & NS \\
\hline III & IV & 33 & 13 & 39,4 & 21 & 4 & 19,0 & 2,478 & 0,290 & NS \\
\hline
\end{tabular}

placebo (hipromelose). Os resultados mostram que apenas houve diferença significativa entre os grupos que foram tratados com cetorolac e hipromelose $(\mathrm{p}<0,05)$ (Tabela 1$)$. Não houve diferença estatisticamente significativa entre cetorolac, flurbiprofeno e diclofenaco.

No estudo da suficiência da dilatação pupilar (> 6mm) obtiveram-se as seguintes proporções: 94,44\% (GI), 73,08\% (GII), 87,88\% (GIII) e 71,43\% (GIV), revelando-se diferença estatisticamente significativa entre o grupo I e os outros três grupos $(p<0,05)$. E a estatística exploratória permite que se perceba a dimensão da variação deste desempenho dentro de cada grupo individualmente (Gráfico 1).

Avaliando a freqüência de miose intra-operatória, encontraram-se 16,67\% GI, 46,15\% GII, 45,45\% GIII e 47,62\% GIV. E, através do teste $\chi^{2}$, demonstra-se diferença significativa entre o GI e os grupos GII, GIII e GIV ( $p<0,05)$, podendo-se, através da estatística exploratória, constatar menores índices de miose intra-operatória para o GI, em relação aos outros três grupos, que alcançaram índices equivalentes entre si (Gráfico 2).

Quando se questionou sobre a utilização de adrenalina durante a operação, foram encontradas as seguintes proporções: 27,77\% (GI), 23,07\% (GII), 21,21\% (GIII) e 28,57\% (GIV), não se observando diferença estatisticamente significativa entre os grupos $(P=0,898)$.

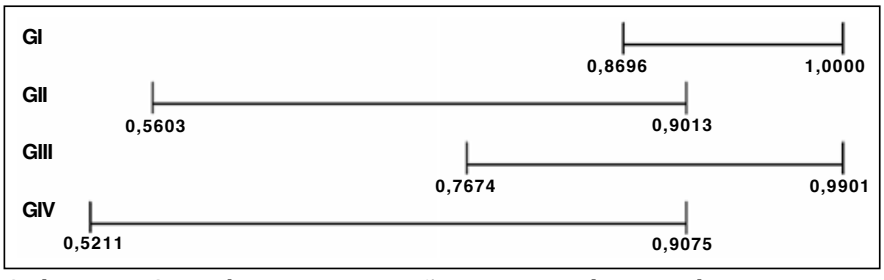

Gráfico 1 - Suficiência da dilatação pupilar pré-operatória - Intervalo de confiança $(p=0,05$ e $z=1,96)$

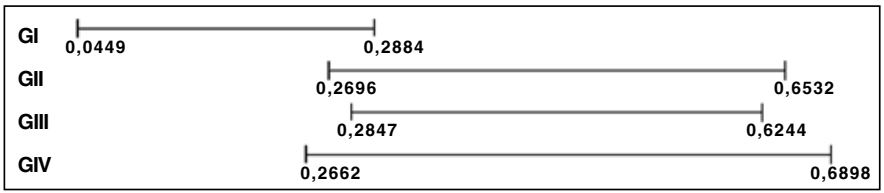

Gráfico 2 - Ocorrência de miose intra-operatória - Intervalo de Confiança $(p=0,05$ e $z=1,96)$
Ademais, com o uso do carbacol, ao final da cirurgia, obteve-se $100 \%$ de miose (resultado positivo) em todos os casos, com exceção do flurbiprofeno, em que houve 7,69\% de não-miose após seu uso (2 em 26 pacientes).

\section{DISCUSSÃO}

Os achados são concordantes com Gupta et al. que compararam o uso de cetorolac por via oral e o placebo, mostrando diferença estatisticamente significativa na manutenção da dilatação pupilar ${ }^{(7)}$. Entretanto, aquele estudo mostrou diferença significante entre o uso de flurbiprofeno e do placebo, enquanto que o presente estudo mostra diferenças percentuais, não significantes.

Outra vez os resultados apresentados por Gupta et al. condizem com este estudo, na apresentação de diferença não significativa entre flurbiprofeno e cetorolac para a manutenção da midríase ${ }^{(7)}$.

O diclofenaco exibiu melhor desempenho percentual (não significativo) do que o flurbiprofeno, com o que concordam Sachdev et $\mathrm{al}^{(6)}$. Neste estudo a diferença entre diclofenaco e placebo não se confirma estatisticamente

As respostas ao questionário pré-operatório indicam que o grupo exposto ao cetorolac apresentou índices de midríase considerados para a realização da cirurgia e ocorrência de miose intra-operatória em proporções significativamente melhores do que nos outros três grupos. Para o uso de adrenalina, não foram encontradas diferenças significativas entre os quatro grupos.

Com o uso do cetorolac, diclofenaco e hipromelose (placebo) foi possível obter $100 \%$ de miose, como resposta positiva ao carbacol, enquanto a resposta positiva do flurbiprofeno ao carbacol foi de $92,31 \%{ }^{(6)}$. Esta resposta ao carbacol é dado de relevância, porque evidencia que, embora haja manutenção da midríase per-operatória com os antiprostaglandínicos, essa ação é reversível, o que facilita a obtenção da miose ao final da cirurgia de catarata.

Os resultados evidenciam que o cetorolac foi mais eficiente, dentro das condições de teste neste estudo, que as demais substâncias avaliadas em relação à manutenção da dilatação pupilar, suficiência da midríase e freqüência de miose intraoperatória. Os resultados encontrados para flurbiprofeno e 
822 Estudo comparativo da eficácia de colírios antiinflamatórios não esteróides na manutenção da dilatação pupilar durante a cirurgia de catarata

diclofenaco são semelhantes aos encontrados por outros autores, não obstante não havendo diferença estatisticamente significativa entre estes e o placebo.

\section{ABSTRACT}

Background and Purpose: Nonsteroidal antiinflammatory drugs, with antiprostaglandin effect, have been used in the preoperative period of cataract surgery for maintenance of mydriasis during the surgery. Unfortunately, the ideal antiprostaglandin has yet to be introduced. This study is a transversal cut, randomized, placebo-group and double-masked clinical trial comparing the efficacy of ketorolac tromethamine, flurbiprofen sodium and diclofenac sodium with a placebo (hypromellose) in maintaining mydriasis during surgery. Methods: One hundred sixteen patients, undergoing extracapsular cataract extraction with intraocular lens implantation, were randomly assigned to four groups, which had the same routine for dilation and use of antiprostaglandins. Group I received ketorolac tromethamine; group II, flurbiprofen; group III, diclofenac and group IV, control group, hypromellose. The pupillary data were obtained through an objective questionnaire answered by each surgeon after surgery. Results: Group I was statistically different from placebo regarding maintenance of dilation during surgey. The difference was statistically significant between GI and the other regarding sufficient mydriasis for the beginning of surgery and intraoperative miosis. Flurbiprofen and diclofenac were not statistically different and also when they were compared to the placebo as regards the commented above parameters. Conclusions: Ketorolac tromethamine was more effective than other antiprostaglan- dins and placebo considering the tested parameters. Flurbiprofen and diclofenac were better than placebo considering percentual values, with no statistical difference. An increase in the number of our sample as well as other multicentric studies will help to establish a definitive role for antiprostaglandins in maintaining mydriasis during surgery.

Keywords: Ophthalmic solutions; Cataract extraction; Pupil/ drug effects; Antiinflammatory agents, nonsteroidal/therapeutic use; Efficacy; Comparative study; Adult

\section{REFERÊNCIAS}

1. Camras CB, Miranda OC. The putative role of prostaglandins in surgical miosis. Prog Clin Biol Res 1989;312:197-210.

2. Chaudhary KP, Sofat BK. Preoperative topical flurbiprofen-Na+ in extracapsular lens extraction role in maintaining intraoperative pupillary dilatation. Indian J Ophthalmol 1992;40;109-14.

3. Drews RC, Katsev DA. Ocufen and pupillary dilation during cataract surgery. J Cataract Refract Surg 1989;15:445-8.

4. Heinrichs DA, Leith AB. Effect of flurbiprofen on the maintenance of pupillary dilation during cataract surgery. Can J Ophthalmol 1990;25:239-42.

5. Keates RH, McGowan KA. Clinical trial of flurbiprofen to maintain pupillary dilation during cataract surgery. Ann Ophthalmol 1984;16:919-21.

6. Sachdev MS, Singh K, Talwar D, Gupta SK, Dada VK. Comparative efficacy of diclofenac and flurbiprofen for maintenance of pupillary dilatation during cataract surgery. Ophthalmic Surg 1994;25:92-4.

7. Gupta VP, Dhaliwal U, Prasad N. Ketorolac tromethamine in the maintenance of intraoperative mydriasis. Ophthalmic Surg Lasers 1997;28:731-8.

8. Sachdev MS, Mehta MR, Dada VK, Jain AK, Garg SP, Gupta SK. Pupillary dilatation during cataract surgery - relative efficacy of indomethacin and flurbiprofen. Ophthalmic Surg 1990;21:557-9.

9. Holmes JM, Jay WM. The effect of preoperative flurbiprofen on miosis produced by acetylcholine during cataract surgery. Am J Ophthalmol 1991;111: 735-8.

10. Sé DCS, Marback RL. Midríase em cirurgias de catarata. Arq Bras Oftalmol 1995;58:361-3

\title{
ABO ELETRÔNICO
}

\author{
Novo site
}

\section{Acesso: http://www.abonet.com.br}

